\title{
Effectively Characterize Planar-view FinFET Semiconductor Device Etch Uniformity by Introducing Diffraction Contrast in STEM Imaging
}

\author{
Wayne W. Zhao ${ }^{1}$ \\ 1. Physical Failure Analysis, Center for Complex Analysis, Characterization Group, Fab8, \\ GLOBALFOUNDRIES, Malta, NY.
}

In scanning transmission electron microscopy (STEM), two types of imaging modes are primarily employed, which are complimentary to each other, namely, the high-angle annular dark-field (HAADF) STEM, and the low-angle annular dark-field (LAADF) STEM. HAADF-STEM and LAADF-STEM modes record different portions of the STEM signals. HAADF signals are primarily collected by the outer ring of the STEM detector, which forms a relatively high angle to the electron beam, and close to the TEM sample, in Z-direction. This optical configuration minimizes diffraction contrast, and enables HAADF-STEM for true Z-contrast, i.e., its contrast is closely proportional to the average atomic weight of the region being probed. LAADF-STEM, on the contrary, mainly utilizes the inner small ring detector, which is below the HAADF-detector and at a smaller angle (than the HAADF) to the electron beam path. Diffraction contrast can be easily enhanced in the LAADF-STEM, e.g., by a large camera length to toggle between the HAADF-STEM and LAADF-STEM. Since STEM imaging is usually coupled with x-ray energy dispersive spectroscopy (XEDS) and electron energy loss spectroscopy (EELS), HAADF-STEM is more frequently employed than LAADF-STEM, to fully take advantage of the pure Z-contrast, for compositional accuracy. However, in certain special applications, strong diffraction contrast under STEM can help to better delineate features of interests than the Z-contrast by HAADF-STEM. The example below demonstrated one such effectiveness by introducing diffraction contrast in STEM during a planar-view characterizations, i.e., the TEM lamella is parallel to the wafer surface / along Si (100) crystal plane, of etch uniformity for FinFET device integration in waferfoundries.

Semiconductor devices with nanometer-scale transistors built on Integrated Chips (IC) are basically a complex miniature stack with composites of various material properties, e.g., semiconductors, interconnects, and dielectrics. Improving uniformity within the same wafer and across wafers during various steps of process is a constant theme in wafer foundries, to ensure continuous yield enhancement. With a large field of view in addition to the highest spatial resolution, planar-view TEM/STEM helps to inspect extents of uniformity across multiple transistors, in one TEM lamella. For example, to monitor the uniformity and effectiveness in selective etch at the intersections of Fins and Gates in a FinFET device is a critical feedback, necessary for tool qualification, centering new masks, and tuning recipes for continuous improvement of process (CIP). Figure-1a is a simplified TCAD cartoon, illustrating the complex nanometer transistor device structure. Underetch of the Fin-Gate intersections will induce socalled "PC footing" or "PC skirt" defects, which increases chances for out-of-specification PC formation in the down-stream process and yield impact due to premature device fails. Fins are crystalline Si, dummy gates are polycrystalline / amorphous Si covered with a SiO spacer and a SiN hard mask. For severe underetch initially, planar-view by TEM is good enough to discern the corners of Fins and Gates, as in Fig.-1b. With subtle residuals during fine-tuning etch recipes, even HAADF-STEM could not yield enough contrast, since average atomic-weights of $\mathrm{Si}+\mathrm{O}$ and $\mathrm{Si}+\mathrm{N}$ are similar to $\mathrm{Si}$. However, by introducing diffraction contrast, LAADF-STEM clearly reveals the true boundary / corners of the crystalline Si Fins versus amorphous $\mathrm{SiO} / \mathrm{SiN}$. It is evident that LAADF-STEM is very effective, as 
shown in Figures-1c 1h, for TEM, HAADF-STEM, and LAADF-STEM, and XEDS of the same site. Zoom-in lattice imaging can always discern the intersection, as in Figures-1i 1k, one example with features decorated with a HfO. However, lattice imaging on small lattice spacing of Si-100 covered by thick amorphous layers of the $\mathrm{SiO} / \mathrm{SiN} / \mathrm{HfO}$ is very time-consuming, e.g., one has to fine-tune the crystal zone Fin by Fin, which jeopardizes the needs for the quickest turnaround in high-volume manufacturing. Planar-view by LAADF-STEM with diffraction contrast is still simple and straightforward. Therefore, it is recommended, as a best practice, to quickly discern etch uniformity in FinFET from a large field of view / sampling window [4].

\section{References:}

[1] W. Zhao, M. Gribelyuk, Microscopy \& Microanalysis 22 (S3) (2016), p. 334.

[2] W. Zhao et al, Microscopy \& Microanalysis 21 (S3) (2015), p. 1036.

[3] W. Zhao et al, Microscopy \& Microanalysis 20 (S3) (2014), p. 362.

[4] Thanks go to Dong-Ick Lee for the TCAD cartoon, and to Albert Amann-III, Travis Mitchell, and Shaun Alvarez of GLOBALFOUNDRIES Fab8 for their excellence in TEM sample-preparations, and Fab8 Management, especially to Edward Crawford for proof-reading, and Legal teams for supporting the publication clearance.
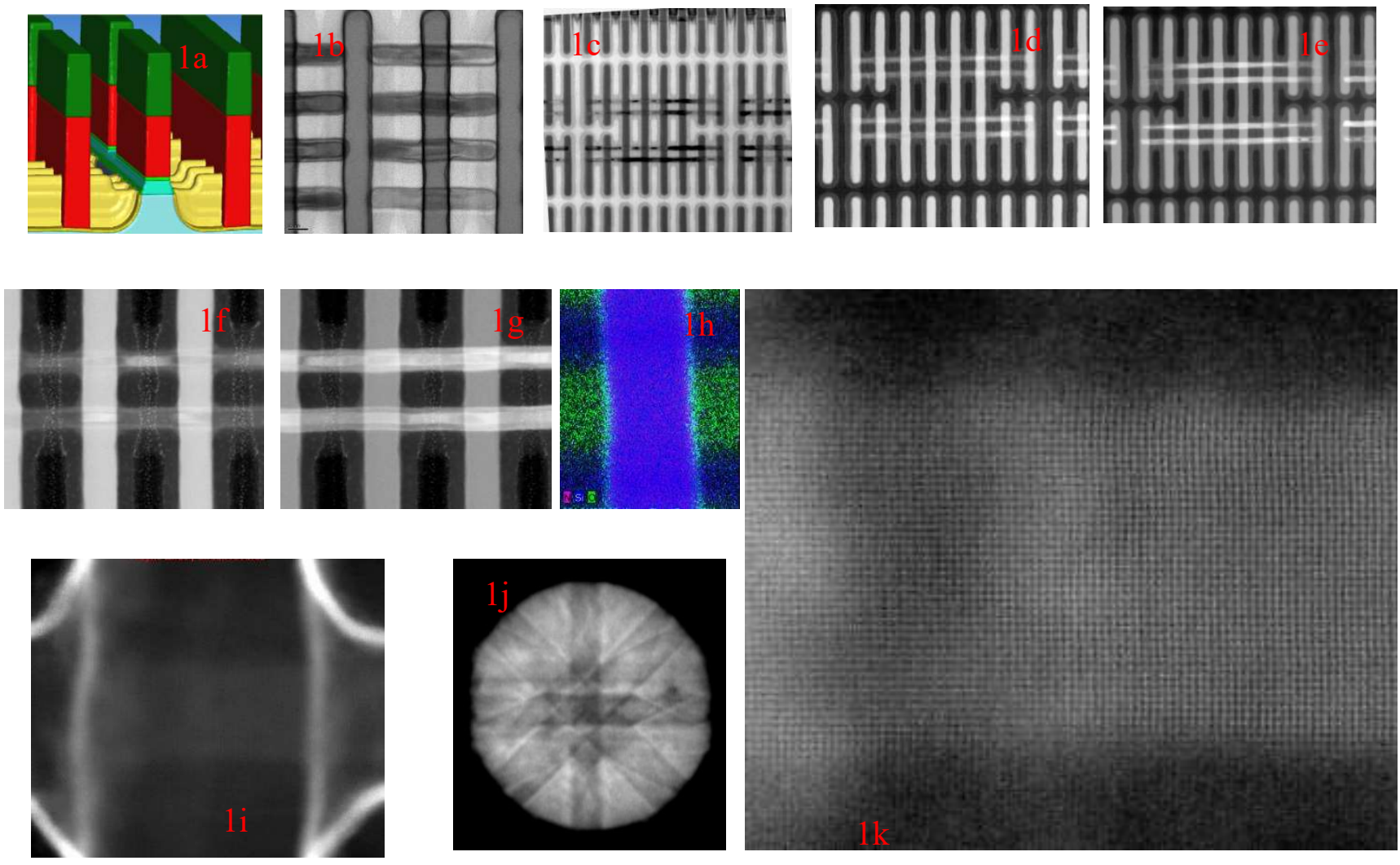

Figure 1. (a) TCAD cartoon; (1b 1c) top-down TEM; (1d and 1f) HAADF-STEM; (1e and 1g) LAADF-STEM; (1h) overlay of XEDS mapping; (1i and 1k) Low- / high-Mag STEM lattice imaging for Si-100, and (1j) its corresponding diffraction / Kikuchi pattern. 